\title{
Induced pluripotent stem cells: reprogrammed without a trace
}

"...technological advances driving the science of induced pluripotency will be increasingly essential for realizing the opportunities of patient-specific, cell-based treatments, thereby translating the discoveries of today into the practice of tomorrow."

Nuclear reprogramming through triggered cellular dedifferentiation offers a revolutionary framework to derive pluripotent stem cells from somatic tissue, independently of an embryo source. The emerging technology demonstrates that ectopic expression of stemness-related genes is sufficient to reset parental cell fate, unlocking the potential of unlimited patient-specific regenerative therapies. Thereby, discovery science has overcome restrictions inherent to embryonic derivation enabling access to a genuine, autologous, pluripotent cell population. Nuclear reprogramming technology must, however, avoid permanent transgenic modifications to establish a safe tool amenable for clinical translation.

Originally, somatic cell nuclear transfer established the concept of genomic intervention to reset cell fate. Transferring the nucleus of an adult cell into the cytoplasmic environment of a host oocyte revealed genetic malleability to reacquire atavistic developmental competency [1,2]. Ensuing nuclear-cytosol interactions catalyze the epigenetic regulation that reverses the differentiation state of the parental nucleus to achieve genetic reprogramming, and recapitulate the embryonic ground state $[3,4]$. The robustness of this process is further evident by successful xenogenic combinations of adult nuclei with primitive cytoplasm components, revealing phylogenic conservation of a permissive gene-environment interface [5]. Recognition that the genetic makeup of 'terminally differentiated' tissue is fully reversible to the pluripotent state paved the way towards embryo-independent engineering, recently implemented through high-throughput candidate-based gene approaches.

Discovery of induced pluripotency was achieved through exposure of discrete sets of qualified pluripotent genes that collectively recapitulates the requirements necessary to reset the phenotypic fate of an ordinary parental source [6]. Delivery of prototypic stemness-related genes
OCT-3/4, SOX2, KLF4 and c-MYC through stable genomic integration reprogrammed adult somatic cells and promoted re-acquisition of embryonic traits, such as germline transmission, previously unique to bona fide embryonic stem cells [7]. Resulting induced pluripotent stem cells (iPS) have met multiple levels of pluripotent stringency, unlike any other adult-derived cytotype, with anachronic cellular features that recapitulated primitive morphological marks of stem cells, expressed biomarkers consistent with authentic pluripotency, produced teratoma in vivo, contributed to chimeric offspring, and gave rise to germline transmission or completely iPS-derived embryos using tetraploid aggregation [8,9]. Alternative combinations of distinctive gene sets expressed at different stoichiometry have effectively reprogrammed a host of diverse somatic cell types to validate the robustness of multiple platforms with different transgenic technologies, suggesting redundant mechanisms that can be reactivated by nonexclusive inductors [10-18].

Retroviral and lentiviral approaches offered the initial methodology that launched the field, and established the technological basis of nuclear reprogramming with rapid confirmation across integrating vector systems $[6,7,10-18]$. The risk of oncogenic genes and insertional mutagenesis inherent to stable genomic integration triggered, in turn, the search for the next generation of iPS production. Because the retroviral-based vector systems have built-in sequences that silence the process of transcription upon pluripotent induction, persistent exposure to ectopic gene expression was temporally restricted indicating that successful self-maintaince of the pluripotent state was possible without long-term transgene expression. Thereby, systems were designed for transient production of stemness-related genes without integration into the genome. The first proof-of-principle was achieved by nonintegrating viral vector systems, such as adenovirus [19], and confirmed by

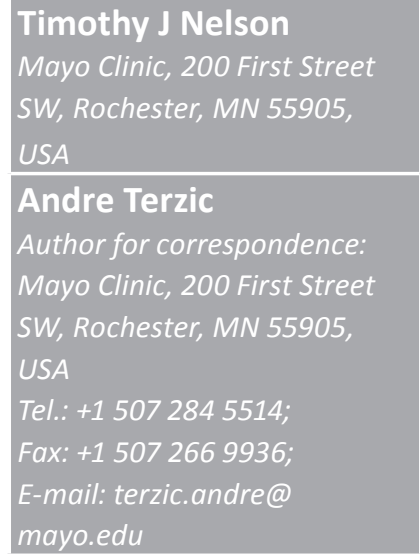

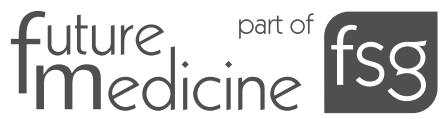


repeated exposure to extrachromosomal plasmidbased transgenes [20]. Importantly, these reports demonstrated that expression of stemness-related factors was required for only a limited timeframe until progeny developed autonomous selfrenewal, establishing nuclear reprogramming as a bioengineered process that resets a sustainable pluripotent cell fate independent of permanent genomic modifications. However, the inherent inefficiency of nonintegrated technologies has hindered broader applicability.

\section{"Although the full impact of stem cell-based regenerative medicine will not be realized for decades, it is anticipated that the latest advances will lead to rapid adoption and Investigational New Drug applications for diseases that require comprehensive tissue replacement."}

The latest innovation that advances iPSbased technology towards clinical applications has recently been reported in which nonviral approaches are capable of high-efficiency iPS production $[21,22]$. These newest approaches are dependent on short sequences of mobile genetic elements that can be used to integrate transgenes into host-cell genomes and provide a genetic tag to 'cut and paste' flanked genomic DNA sequences. The piggyBac (PB) system couples enzymatic cleavage with sequence specific recognition using a transposon-transposase interaction to ensure high efficiency removal of flanked DNA without residual footprint. Importantly, this technology achieves a traceless transgenic approach in which non-native genomic sequences that are transiently required for nuclear reprogramming can be removed upon induction of pluripotency. Using the PB transposition system with randomly integrated stemness-related transgenes, Nagy and colleagues have demonstrated that disposal of ectopic genes could be efficiently regulated upon induction of self-maintaining pluripotency according to expression of the transposase enzyme without infringement on genomic stability [22] This state-of-the-art system is uniquely qualified to allow safe integration and removal of ectopic transgenes, improving the efficiency of iPS production. Alternatively, the security of unmodified genomic intervention can be achieved with nonintegrating episomal vectors [23]. Collectively, these recent strategies accelerate the discovery science of regenerative medicine bringing it a step closer to clinical applicability by allowing genetically unmodified progenitor cells to acquire the capacity of pluripotency and produce unlimited, autologous replacement tissues for patients with degenerative diseases.

To date, the therapeutic benefit of iPS-based technology has been tested in three disease models, namely sickle cell anemia [24], Parkinson's disease [25] and hemophilia A [26]. These regenerative applications have utilized lineages prespecified in vitro to repair inherent genetic or acquired degenerative diseases. As differentiation protocols are refined and preclinical disease models are further screened to address the regenerative value of iPS technology, indications for self-derived iPSbased therapy is poised to progressively expand. Stem cell-based technology, including the next generation of genetically unmodified iPS, will be ultimately positioned to augment the armamentarium of biologics offered by transplant medicine, and expand therapeutic strategies available to patients that today are considered to have incurable diseases [27]. To this end, initial studies demonstrate the feasibility of deriving patient-specific iPS free of reprogramming factors using Cre-recombinase excisable viruses [28]. Although the full impact of stem cell-based regenerative medicine will not be realized for decades, it is anticipated that the latest advances will lead to rapid adoption and Investigational New Drug (IND) applications for diseases that require comprehensive tissue replacement [29]. Beyond advances in stem-cell biology leading to discovery and development of novel biologics, delivery systems and methodologies to accurately and safely transplant cell-based products to the site of damaged tissue will further augment the clinical value and applicability of emerging technology [30].

As medical therapy is moving from a palliative approach to a curative paradigm for individual patients, regenerative medicine will become a priority to healthcare transformation. In this regard, technological advances driving the science of induced pluripotency will be increasingly essential for realizing the opportunities of patient-specific, cell-based treatments, thereby translating the discoveries of today into the practice of tomorrow.

Financial \& competing interests disclosure

The authors have no relevant affiliations or financial involvement with any organization or entity with a financial interest in or financial conflict with the subject matter or materials discussed in the manuscript. This includes employment, consultancies, honoraria, stock ownership or options, expert testimony, grants or patents received or pending, or royalties.

No writing assistance was utilized in the production of this manuscript. 


\section{Bibliography}

1 Campbell KHS, McWhir J, Ritchie WA et al.: Sheep cloned by nuclear transfer from a cultured cell line. Nature 380, 64-66 (1996).

2 Wilmut I, Schnieke AE, McWhir J et al.: Viable offspring derived from fetal and adult mammalian cells. Nature 385, 810-813 (1997).

3 Hochedlinger K, Jaenisch R: Nuclear reprogramming and pluripotency. Nature 441, 1061-1067 (2006).

4 Armstrong L, Lako M, Dean W et al: Epigenetic modification is central to genome reprogramming in somatic cell nuclear transfer. Stem Cells 24, 805-814 (2006).

5 Beyhan Z, Iager AE, Cibelli JB: Interspecies nuclear transfer: implications for embryonic stem cell biology. Cell Stem Cell 1, 502-512 (2007).

6 Takahashi K, Yamanaka S: Induction of pluripotent stem cells from mouse embryonic and adult fibroblast cultures by defined factors. Cell 126, 663-676 (2006).

7 Okita K, Ichisaka T, Yamanaka S: Generation of germline-competent induced pluripotent stem cells. Nature 448, 313-317 (2007).

8 Maherali N, Hochedlinger K: Guidelines and techniques for the generation of induced pluripotent stem cells. Cell Stem Cell 3, 595-605 (2008).

9 Smith KP, Luong MX, Stein GS: Pluripotency: toward a gold standard for human ES and iPS cells. J. Cell Physiol. doi:10.1002/jcp.21681 (2009) (Epub ahead of print)

10 Yu J, Vodyanik MA, Smuga-Otto K et al.: Induced pluripotent stem cell lines derived from human somatic cells. Science 318, 1917-1920 (2007).

11 Aasen T, Raya A, Barrero MJ et al:: Efficient and rapid generation of induced pluripotent stem cells from human keratinocytes. Nat. Biotechnol. 26, 1276-1284 (2008).
12 Aoi T, Yae K, Nakagawa M et al.: Generation of pluripotent stem cells from adult mouse liver and stomach cells. Science 321, 699-702 (2008).

13 Nakagawa M, Koyanagi M, Tanabe K et al.: Generation of induced pluripotent stem cells without Myc from mouse and human fibroblasts. Nat. Biotechnol. 26, 101-106 (2008).

14 Huangfu D, Osafune K, Maehr R et al.: Induction of pluripotent stem cells from primary human fibroblasts with only Oct 4 and Sox2. Nat. Biotechnol. 26, 1269-1275 (2008).

15 Eminli S, Utikal J, Arnold K et al.: Reprogramming of neural progenitor cells into induced pluripotent stem cells in the absence of exogenous Sox 2 expression. Stem Cells 26, 2467-2474 (2008).

16 Kim JB, Zaehres H, Wu G et al:: Pluripotent stem cells induced from adult neural stem cells by reprogramming with two factors. Nature 454, 646-650 (2008).

17 Hanna J, Markoulaki S, Schorderet P et al.: Direct reprogramming of terminally differentiated mature B lymphocytes to pluripotency. Cell 133, 250-264 (2008).

18 Feng B, Jiang J, Kraus P et al.: Reprogramming of fibroblasts into induced pluripotent stem cells with orphan nuclear receptor Esrrb. Nat. Cell Biol. 11, 197-203 (2009).

19 Stadtfeld M, Nagaya M, Utikal J et al.: Induced pluripotent stem cells generated without viral integration. Science 322 , 945-949 (2008).

20 Okita K, Nakagawa M, Hyenjong $\mathrm{H}$ et al.: Generation of mouse induced pluripotent stem cells without viral vectors. Science 322, 949-953 (2008).

21 Kaji K, Norrby K, Paca A et al.: Virus-free induction of pluripotency and subsequent excision of reprogramming factors. Nature doi:10.1038/nature07864 (2009) (Epub ahead of print).
22 Woltjen K, Michael IP, Mohseni P et al: piggyBac transposition reprograms fibroblasts to induced pluripotent stem cells. Nature doi:10.1038/nature07863 (2009) (Epub ahead of print).

$23 \mathrm{Yu}$ J, Hu K, Smuga-Otto K et al.: Human induced pluripotent stem cells free of vector and transgene sequences. Science doi:10.1126/ science.1172482 (2009) (Epub ahead of print).

24 Hanna J, Wernig M, Markoulaki S et al.: Treatment of sickle cell anemia mouse model with iPS cells generated from autologous skin. Science 318, 1920-1923 (2007).

25 Wernig M, Zhao JP, Pruszak J et al.: Neurons derived from reprogrammed fibroblasts functionally integrate into the fetal brain and improve symptoms of rats with Parkinson's disease. Proc. Natl Acad. Sci. USA 105, 5856-5861 (2008).

26 Xu D, Alipio Z, Fink LM et al.: Phenotypic correction of murine hemophilia A using an iPS cell-based therapy. Proc. Natl Acad. Sci. USA 106, 808-813 (2009).

27 Nelson TJ, Behfar A, Terzic A: Strategies for therapeutic repair: the "R3" regenerative medicine paradigm. Clin. Transl. Sci. 1, 168-171 (2008).

28 Soldner F, Hockemeyer D, Beard C et al.: Parkinson's disease patient-derived induced pluripotent stem cells free of viral reprogramming factors. Cell doi: $10.1016 / \mathrm{j}$. cell.2009.02.013 (2009) (Epub ahead of print).

29 Nelson TJ, Behfar A, Terzic A: Stem cells: biologics for regeneration. Clin. Pharmacol. Ther. 84, 620-623 (2008).

30 Bartunek J, Sherman W, Vanderheyden M et al: Delivery of biologics in cardiovascular regenerative medicine. Clin. Pharmacol. Ther. doi:10.1038/clpt.2008.295 (2009)

(Epub ahead of print). 